American Journal of Primatology 47:133-151 (1999)

\title{
Geographic Variation in the Calls of Wild Chimpanzees: A Reassessment
}

\author{
J. C. MITANI*, K. L. HUNLEY, AND M. E. MURDOCH \\ Department of Anthropology, University of Michigan, Ann Arbor
}

Male chimpanzees produce a species-typical call, the pant hoot, to communicate to conspecifics over long-distances. Calls given by males from the well-known Gombe and Mahale populations typically consist of four different phases: an introduction, build-up, climax, and let-down. Recent observations suggest that chimpanzees living in the Kibale National Park, Uganda, consistently give calls that lack a build-up and are thus qualitatively distinguishable acoustically from those made by other East African conspecifics. We analyzed additional recordings from Mahale and Kibale to re-examine geographic variation in chimpanzee calls. Results indicate that males from both sites produce pant hoots containing all four parts of the call. Calls made by chimpanzees from the two populations, however, differ in quantitative acoustic measures. Specifically, males at Kibale initiate their calls with significantly longer elements and buildup over briefer periods at slower rates than individuals from Mahale. Kibale males also deliver acoustically less variable calls than chimpanzees at Mahale. Although climax elements do not differ between populations in any single acoustic feature, discriminant function analysis reveals that acoustic variables can be used in combination to assign calls to the correct population at rates higher than that expected by chance. Ecological factors related to differences in habitat acoustics, the sound environment of the local biota, and body size are likely to account for these observed macrogeographic variations in chimpanzee calls. Am. J. Primatol. 47:133-151, 1999. ( 1999 Wiley-Liss, Inc.

\section{Key words: chimpanzee; vocal communication; geographic variation}

\section{INTRODUCTION}

Variation is a key feature of the living world [Darwin, 1859], and studies consistently reveal acoustic variability in the calls produced by nonhuman primates. Early field research provided ample documentation of interspecific and interindividual differences in vocalizations [e.g., Struhsaker, 1970; Marler \& Hobbett, 1975; Marshall \& Marshall, 1976; Waser, 1977], while more recent investigations have established that calls emitted by the same individual in dis-

Contract grant sponsor: NSF; Contract grant numbers: SBR-9253590, BNS-9021682; Contract grant sponsor: Harry Frank Guggenheim Foundation; Contract grant sponsor: National Geographic Society; Contract grant sponsor: Japanese Ministry of Education, Science, and Culture.

*Correspondence to: John Mitani, Department of Anthropology, University of Michigan, Ann Arbor, Michigan 48109. E-mail: mitani@umich.edu

Received 10 November 1997; revision accepted 10 July 1997 


\section{4 / Mitani et al.}

tinct behavioral contexts differ in subtle acoustic features [e.g., Seyfarth et al., 1980; Gouzoules et al., 1984; Zuberbuler et al., 1997].

In contrast to the abundant field evidence of species, individual, and withinindividual variation in calls, relatively few data exist on population differences in primate vocal behavior [Green, 1975; Maeda \& Masataka, 1987; Mitani et al., 1992]. Between-population variation in primate calls is of special interest insofar as it bears on unresolved evolutionary and behavioral problems. For example, information on population variation in vocalizations complements traditional morphological and molecular data used to infer phylogenetic relationships among organisms [e.g., Hodun et al., 1981; Mitani, 1987]. In addition, population variability in vocal behavior provides evidence regarding the mechanisms underlying call development. Since one correlate of the vocal learning process in humans and animals is the formation of local dialects [e.g., Marler \& Tamura, 1964], dialectal variation can be employed to evaluate the role learning plays in the acquisition of calls [Kroodsma, 1982].

Chimpanzees are a particularly appropriate species to investigate geographic variation in vocal behavior. Recent field research suggests that the acoustic structure of pant hoots, the species-typical, long distance calls produced by chimpanzees [Marler \& Hobbett, 1975], varies between populations. Our own studies indicate that the calls made by males from the well-known Gombe and Mahale populations differ acoustically [Mitani et al., 1992; Mitani \& Brandt, 1994]. Clark Arcadi [1996] has recently extended these results by providing suggestive data that males from an additional population of chimpanzees living in the Kanyawara area of the Kibale National Park, Uganda, produce qualitatively different pant hoots compared with individuals at Gombe and Mahale. Clark Arcadi [1996] interpreted this finding as consistent with the hypothesis that vocal development in chimpanzees involves learning.

The results and conclusions presented by Clark Arcadi [1996] are of considerable interest yet difficult to evaluate empirically and theoretically. First, his comparison was based on limited data from Kibale, with a median number of only four calls from seven individuals included in the sample $(\overline{\mathrm{X}}=12$ calls per individual; S.D. = 20). Second, it is not clear whether the geographic differences reported by Clark Arcadi [1996] are relevant to the question of vocal learning in chimpanzees. To assess the causes and biological significance of geographic variation in calls, it is important to distinguish between variability that occurs on a macrogeographic or regional scale and that which takes place microgeographically or locally [see Marler, 1960; Nottebohm, 1969; Krebs \& Kroodsma, 1980; Conner, 1982; Mundinger, 1982]. Macrogeographic or regional variation refers to differences in vocal behavior between populations that are separated by long distances, and as a result, do not typically mix. These macrogeographic variations in calls are often due to ecological factors such as differences in habitat acoustics [Wiley \& Richards, 1982], the sound environment of the local biota [Marler, 1957; Sorjonen, 1986], or anatomical differences between animals [Bowman, 1979]. Microgeographic or local variation, generally referred to as "local" dialects [Krebs \& Kroodsma, 1980; Conner, 1982; Mundinger, 1982], occurs between neighboring populations or populations of potentially interbreeding individuals. In contrast to regional variations, local dialects are not easily attributed to ecological adaptation to different environments or genetic differences between populations but are instead a likely by-product of the vocal learning process [Marler, 1960; Nottebohm, 1969; Kroodsma, 1982; Mundinger, 1982]. The dis- 
Geographic Variation in Chimpanzee Calls / 135

tinction between regional and local variation in vocal behavior suggests that a parsimonious explanation for differences in the calls of extant chimpanzee populations living in widely separated areas of present-day Tanzania and Uganda need not invoke vocal learning.

In this paper, we re-examine geographic variation in the calls produced by wild chimpanzees. For our analysis, we utilize a sample of calls from chimpanzees living in two widely separated populations: the Mahale Mountains National Park, Tanzania, and the Kibale National Park, Uganda. Our comparison is based on the largest sample heretofore assembled, allowing us to control for individual acoustic differences in calls. Our results confirm that readily apparent quantitative differences exist between the calls produced by males from these two isolated populations, though not precisely in the manner previously described by Clark Arcadi [1996]. We interpret these differences in terms of ecological factors that are known to affect patterns of macrogeographic variation in animal calls.

\section{METHODS}

\section{Study Site, Subjects, and Recordings}

We conducted field work on two populations of eastern or long-haired chimpanzees (Pan troglodytes schweinfurthii) in East Africa. We made tape recordings from chimpanzees at the Mahale Mountains National Park, Tanzania $\left(6^{\circ} \mathrm{S}\right.$ and $30^{\circ} \mathrm{E}$ ) during three periods between December 1989 and June 1990, June and August 1992, and March and August 1994. Subjects included members of the $\mathrm{M}$ unit-group or community living at the Kasoje study site in Mahale (Nishida, 1990]. From 1989-1994, M group fluctuated between 80-90 individuals. Tape recordings from a second population of chimpanzees, the Ngogo community in the Kibale National Park, Uganda $\left[0^{\circ}\right.$ and $30^{\circ}$ E, Ghiglieri, 1984], were made during three additional study periods between June and August 1995, June and December 1996, and June and August 1997. Current data suggest that over 110 individuals live within the Ngogo community (Watts and Mitani unpublished data), placing it slightly larger than $M$ group at the times of study. The two populations lie along the eastern edge of the western rift valley of East Africa and are separated by approximately $700 \mathrm{~km}$.

Chimpanzee calls vary as a function of the age and sex of signalers [Marler $\&$ Hobbett, 1975]. We controlled for these potential sources of variation by limiting our analyses to adult males, individuals who have reached physical and social maturity by 15 years of age [Goodall, 1986; Nishida et al., 1990]. We employed calls from 10 males at Mahale and 12 males at Kibale. Table I shows the identities of the study subjects along with sample sizes of recordings used in the following analyses. The majority of calls from both populations were recorded in feeding, traveling, or resting contexts $($ Mahale $=88 \%$ of all calls; Kibale $=96 \%$ ); calls from the two groups were sampled from each of these three contexts with equal frequency $\left(\chi^{2}=4.99,2 \mathrm{df}, P>0.05\right)$. All tape recordings were made ad libitum during hourly behavioral observation sessions or while following chimpanzees opportunistically at other times. Tapes were recorded with a Sony WMD6C recorder and Sennheiser ME80 microphone.

\section{Description of Pant Hoots}

Pant hoots can consist of four distinct parts (Fig. 1). Calls may begin with a brief introductory phase consisting of relatively long, tonal elements that are 


\section{6 / Mitani et al.}

TABLE I. Study Subjects and Sample Sizes of Calls

\begin{tabular}{lccc}
\hline $\begin{array}{l}\text { Mahale } \\
\text { males }\end{array}$ & $\begin{array}{c}\text { Number of } \\
\text { recordings }\end{array}$ & $\begin{array}{c}\text { Kibale } \\
\text { males }\end{array}$ & $\begin{array}{c}\text { Number of } \\
\text { recordings }\end{array}$ \\
\hline BA & & & 39 \\
BE & 24 & AY & 11 \\
FN & 20 & BF & 48 \\
JI & 34 & BT & 23 \\
LJ & 14 & DO & 21 \\
MA & 13 & HO & 17 \\
NT & 12 & HR & 17 \\
NS & 26 & JR & 30 \\
SU & 41 & LO & 26 \\
TB & 14 & MO & 35 \\
& 12 & MW & 30 \\
& & MZ & 13 \\
\hline
\end{tabular}

emitted at low frequencies and amplitudes. The introduction is characterized by a sequential alternation of relatively high frequency elements followed by lower frequency sounds. The introduction grades into the build-up, a series of relatively short elements typically delivered at faster rates than those heard in the introduction and emitted both on inhalation and exhalation. Build-ups are followed by the climax, the loudest and highest frequency component of the call. Calls may end with a brief let-down phase, whose elements do not appear to differ appreciably from those emitted during the build-up. Male subjects at Mahale use pant hoots selectively to maintain contact with preferred associates and allies over long distances [Mitani \& Nishida, 1993], and Kibale chimpanzees at Ngogo appear to employ these calls in the same fashion over similar distances.

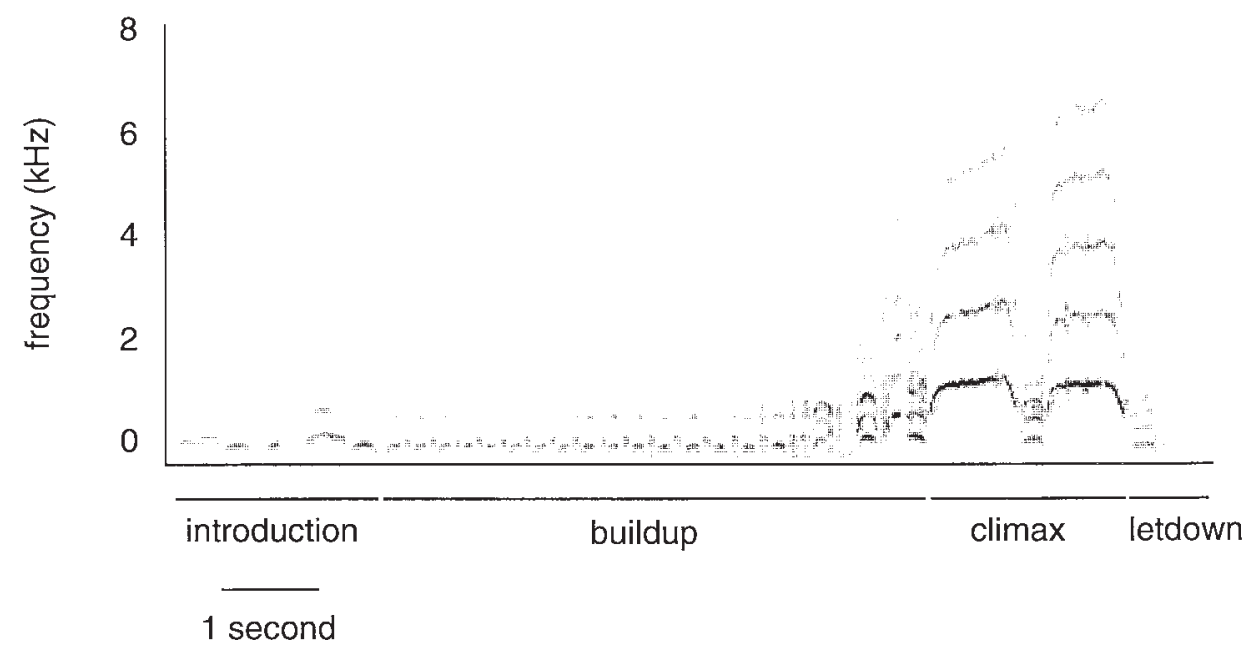

Fig. 1. Audiospectrogram of a representative male chimpanzee pant hoot showing the four distinct phases of the call. The spectrogram was produced using MacRecorder sound analysis software. Analysis range = 11 $\mathrm{kHz}$, frequency resolution $=43 \mathrm{~Hz}$. 
Geographic Variation in Chimpanzee Calls / 137

\section{Acoustic Analyses}

We compared calls given by males from Kibale and Mahale by performing two analyses, one based on a qualitative sorting technique and a second utilizing quantitative measurements of introduction, build-up, and climax elements. Following the previous analysis of Clark Arcadi [1996], we included only those calls that showed clear introduction and climax phases judged spectrographically in the following analyses.

Qualitative sorting. We began by sorting calls into those that contained build-ups and those that lacked these phases by using a qualitative sorting procedure based on visual inspection of audiospectrograms. This process required that we define the build-up phase with sufficient precision that an untrained observer would be able to classify calls. We distinguished build-up phases from introductory portions by their characteristic alternating pattern of tonal, exhaled elements followed by broad-band, noisy, inhaled elements (Fig. 1). This last feature of broad-band, noisy, inhaled elements is a particularly salient and distinguishing feature of most build-ups. By using these observations, we defined the start of the build-up by the single exhaled element that preceded the first distinct, broad-band, noisy inhaled element in the call. Build-ups by this definition start with exhaled elements. Phase endings were defined by the portion of the call that immediately preceded the first exhaled element greater than $500 \mathrm{~Hz}$. Given the considerable heterogeneity in calls, we employed two additional classification rules to handle potentially ambiguous cases. First, we observed that on some occasions one or more barks interrupted a clear build-up phase. In these situations, we included barks as part of the build-up. Second, in some calls exhaled elements consistently broke the " $500 \mathrm{~Hz}$ rule" (see above); by definition, we excluded these elements from the build-up. All calls were qualitatively sorted by a naive observer $(\mathrm{KLH})$ who was originally unaware of the derivation of the individuals and their home populations. Calls of individual males were presented to the observer and sorted together as a group, with individuals from the two populations randomized before classification.

Quantitative measurements. In order to assess quantitatively possible differences between the calls produced by males from Kibale and Mahale, we measured several variables from introduction, build-up, and climax elements. In order to ensure independence of data points, we selected only one introduction, buildup, and climax element from each call for the following analyses.

We examined acoustic features of calls using a digital signal processing program developed for the analysis of animal vocalizations [Beeman, 1996]. Calls were sampled digitally at 20,000 points/second yielding an effective analysis range of $0-8 \mathrm{kHz}$. We measured two acoustic variables from the longest introductory element from each call. We measured element durations directly from their waveforms and obtained frequency values by performing a 1,024 point FFT at the midpoint of each element (frequency resolution $=20 \mathrm{~Hz}$ ). Four additional acoustic variables were measured from the build-up phase and elements contained within it. First, we ascertained the duration of the build-up phase by subtracting the time of the start of the climax phase from the starting time of the first buildup element. Second, we computed a rate of delivery of build-up elements by counting the number of exhaled elements in each call and dividing this by the duration of the build-up phase. For our third and fourth measures, we selected the single exhaled build-up element from the middle of each phase; for calls with an even number of elements, we chose the element immediately preceding the middle of the phase. We then measured the durations of these elements directly from their 


\section{8 / Mitani et al.}

waveforms and their frequencies, derived from 1,024 point FFT's over the midpoints of each element.

Climax elements contain multiple frequency bands that appear to be harmonically related on conventional sound spectrograms and amplitude spectra (Figs. 1, 2c). Most frequency measures were made on the lowest band visible on spectrograms (Figs. 1a, 2c). We measured seven acoustic variables from the single climax element that reached the highest frequency in each call. We began by computing minimum and maximum frequencies from spectrograms of each element after conducting a 512 point Fourier transform (frequency resolution = 39 $\mathrm{Hz}$; Fig. 2a). Climax element durations were measured directly from spectrograms or associated waveforms. From these three acoustic variables, we obtained one additional derived variable, frequency range, by subtracting minimum frequencies from maximum frequencies (Fig. 2a).

We computed average frequencies using 12 equally spaced values across each element. These values were calculated by performing 12 successive 1,024 point Fourier transforms over the length of each signal. The first measurement was made at the start of the signal, with successive measurements made at intervals equal to approximately $1 / 12$ of the duration of the element. Previous analyses indicated that use of an interval equal to one twelfth of calls typically yielded spurious frequency measures of the terminal portions of the longest elements due to low amplitudes at those points [Mitani \& Brandt, 1994; Mitani et al., 1996]. As a result, we moved the last measurement away from climax element endings by employing an interval marginally longer than one-twelfth of the signal ( $=0.087$ call duration).

Two additional variables were computed from the two lowest frequency bands visible from amplitude spectra of calls (Fig. 2b,c). We measured the bandwidth of the lowest frequency band after conducting a 1,024 point FFT over the midpoint of each element. We then went $3 \mathrm{~dB}$ down either side of the spectral peak and subtracted the lower from higher frequency to calculate bandwidths [Fig. 2b; Dunn, 1961]. As noted above, climax elements show multiple frequency bands that appear to be harmonically related on audiospectrograms (Fig. 1). To assess variation in these bands, we employed the 1,024 point FFT's made for bandwidth calculations and computed frequencies and associated amplitudes of the first (F1) and second bands (F2). We used the ratio of the amplitudes of F1 to F2 as an additional acoustic variable. This last measure gives an indication of the amount of spectral energy in the lower part of the frequency spectrum. Since amplitudes are expressed in negative decibels (Fig. 2c), lower values of this ratio indicate greater energy in F1 compared to F2.

\section{Statistical Analyses}

We used the qualitative sorting technique to tally the number of pant hoots that contained build-ups and then calculated the mean percentage of calls containing build-ups for each individual. We compared these mean values between the two populations with a Student's t-test. To satisfy the as-

Fig. 2. Acoustic variables. a: Climax element of pant hoot. Four acoustic variables measured from the lowest frequency band of the element are shown. The spectrogram was produced as in Figure 1. b: Amplitude spectrum of climax element. A 1,024 point FFT taken over the midpoint of the call displays the bandwidth of the lowest frequency band in the element. c: Amplitude spectrum of climax element. A 1,024 point FFT taken over the midpoint of the call shows the amplitudes of the first (F1) and second (F2) frequency bands in the call. The ratio of F1/F2 is used as an acoustic variable. Amplitude spectra were produced with Signal sound analysis software. All figures depicted in a-c are derived from the first climax element shown in Figure 1. 
Geographic Variation in Chimpanzee Calls / 139

a.

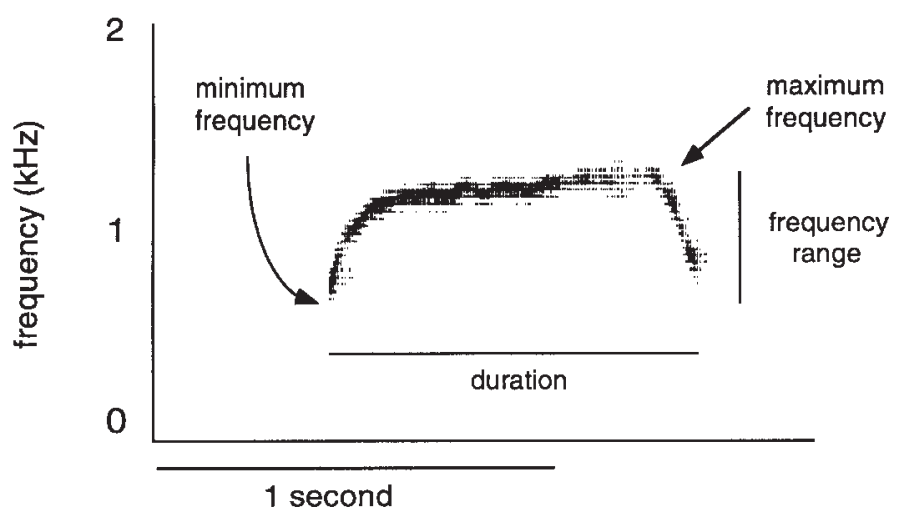

b.

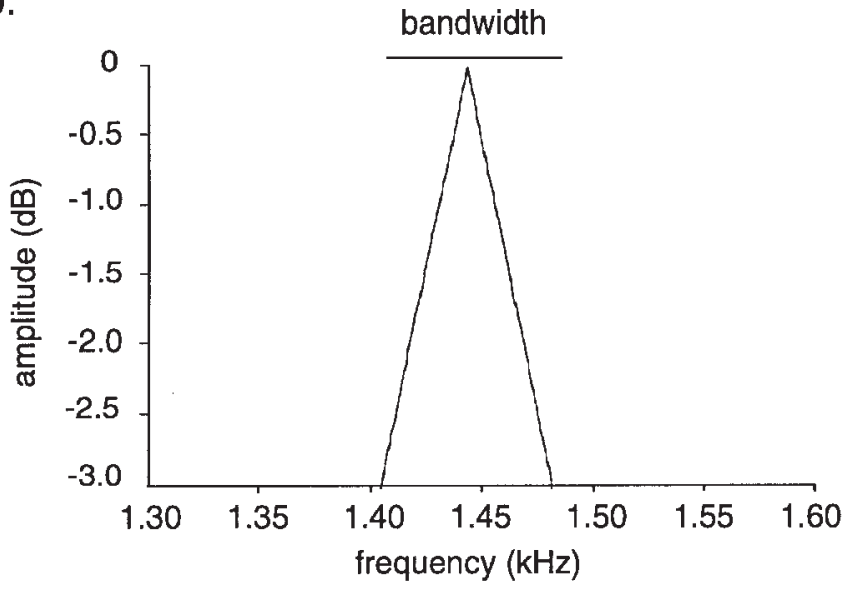

C.

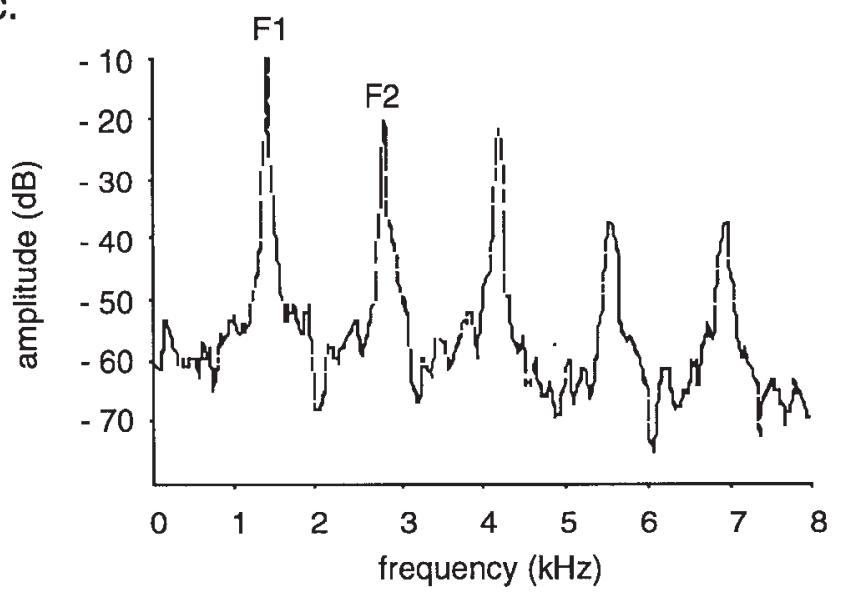

Figure 2. 


\section{0 / Mitani et al.}

sumptions of ANOVA, we used arcsine transformed variables for this analysis [Sokal \& Rohlf, 1995].

We employed uni- and multi-variate parametric statistical tests to examine differences between quantitative measures made from the two populations. We used a nested analysis of variance to compare mean values of acoustic variables measured from the introductory, build-up, and climax phases of each call. Results of the nested ANOVA's provided estimates of variance components attributable to variation between populations, between individuals within populations, and between calls within individuals for each variable [Sokal \& Rohlf, 1995]. All tests were conducted on logarithmically transformed variables. To correct for the increased probability of committing Type I error when making comparisons between populations, we adjusted our criteria of significance downward using a sequential Bonferroni procedure [Holm, 1979]. For k multiple tests, our adjusted alpha levels were set at

$$
\mathrm{P}_{\mathrm{i}} \leq \mathrm{a} /(1+\mathrm{k}-\mathrm{i})
$$

where $\mathrm{a}=0.05$ is the overall experimentwise error rate and $\mathrm{i}$ is the ith sequential test.

For these adjustments, we treated each phase of the pant hoot as a different analysis and considered the number of acoustic variables as the number of comparisons or tests. Thus, for the introduction phase with $\mathrm{k}=2$ acoustic variables, we made our initial adjustment downward to $P<0.025$. Similarly, for the buildup phase with $\mathrm{k}=4$ variables, we began with a level of significance of $P<0.01$, while for the climax phase with $\mathrm{k}=7$ variables, our starting alpha level was $P<$ 0.007. For purposes of presentation, we report all comparisons where $P<0.05$; we consider only those that meet the sequential Bonferroni criteria as significant statistically, however.

We conducted discriminant function analyses as a second means to investigate variation in the calls produced by males from the two populations [Klecka, 1980]. We performed two separate analyses for build-up phases and climax elements. To generate the discriminant functions, we used only those acoustic variables that were shown to differentiate calls from the two populations in the nested ANOVA. Here we set the criteria of significance at $P<$ 0.05 . We withheld a subsample of calls from the calculation of the discriminant functions and used them to cross-validate the classification results of the discriminant analyses. Approximately one half of all available calls were assigned to these subsamples by using a random number generator. Calls from Kibale outnumbered those from Mahale in our sample, and before conducting discriminant analysis computations, we adjusted the prior probabilities of group membership following the algorithm implemented in Systat [Wilkinson et al., 1992].

Finally, we constructed variability profiles to compare within-individual variation in calls produced by males from the two populations [Sokal \& Braumann, 1980]. These profiles were generated by calculating the mean coefficients of variation of males in both populations and then plotting these values for each acoustic variable. We compared the profiles of the two populations with a Student's t-test to investigate whether males from one population produced more variable calls than individuals from the other population. 
Geographic Variation in Chimpanzee Calls / 141

\section{RESULTS}

\section{Qualitative Comparison: Presence and Absence of Build-ups}

Qualitative inspection of audiospectrograms revealed considerable heterogeneity in the production of build-ups by different individuals irrespective of their geographic location. Figure 3 displays representative exemplars of calls in which build-ups are present and absent from males in each of the two populations. Figure 4 shows the mean percentage of calls containing build-ups delivered by each male in the Mahale and Kibale study populations; males from the two populations did not differ in their tendency to emit this portion of the call (Mahale: $\overline{\mathrm{X}}$ $=77 \%$, se $=6$, median $=79 \% ;$ Kibale: $\overline{\mathrm{X}}=66 \%$, se $=8$, median $=75 \% ; \mathrm{t}=0.87, \mathrm{df}$ $=20, P>0.35$ ).

\section{Comparisons of Introduction and Build-up Phases}

Despite the lack of qualitative acoustic differences in the calls of Mahale and Kibale males, easily discerned quantitative differences appear in the introductory and build-up phases of their calls. Kibale males deliver considerably longer introductory elements than Mahale males (Fig. 5a; $\mathrm{F}_{1,20}=22.99, P<0.001$ ). In addition, males at Kibale produce longer build-up elements at slower rates than individuals from Mahale (build-up element durations: $\mathrm{F}_{1,20}=11.70, P<0.005$, Fig. 5c; build-up rates: $\mathrm{F}_{1,20}=8.26, P<0.01$, Fig. $5 \mathrm{~b}$ ). An additional temporal measure of the build-up phase, phase duration, also showed a pronounced and significant difference between the two populations, with Mahale males giving longer build-ups than Kibale individuals (Fig. 5b; $\mathrm{F}_{1,20}=6.91, P<0.025$ ). Frequency measures of introductory and build-up elements did not differ between calls produced by males from the two populations (Fig. 5a,b; nested ANOVA, $P>$ 0.10 for both comparisons). Table II summarizes the preceding results by displaying variance components due to populations, individuals within populations, and calls within individuals for the acoustic variables measured from the introduction and build-up.
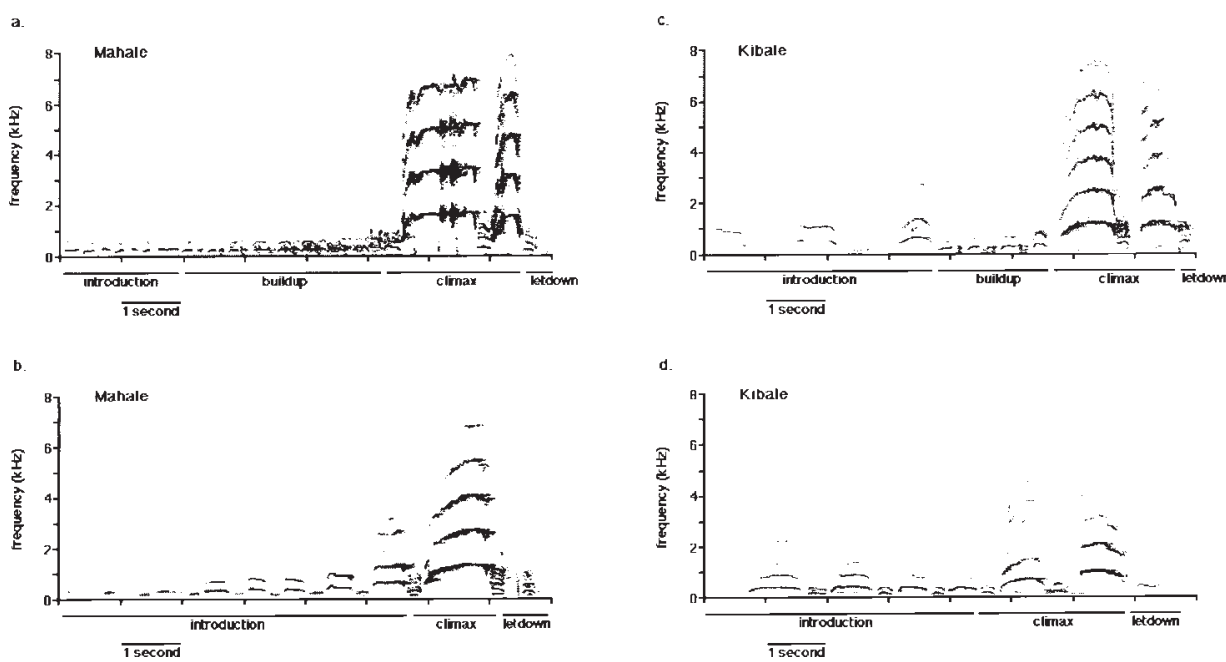

Fig. 3. Audiospectrograms of pant hoots containing and lacking build-up phases. a,c: Complete calls from Mahale and Kibale with build-ups. b,d: Calls from Mahale and Kibale lacking build-ups. Spectrograms were produced with Signal sound analysis software. Analysis range $=8 \mathrm{kHz}$, frequency resolution $=20 \mathrm{~Hz}$. 
142 / Mitani et al.

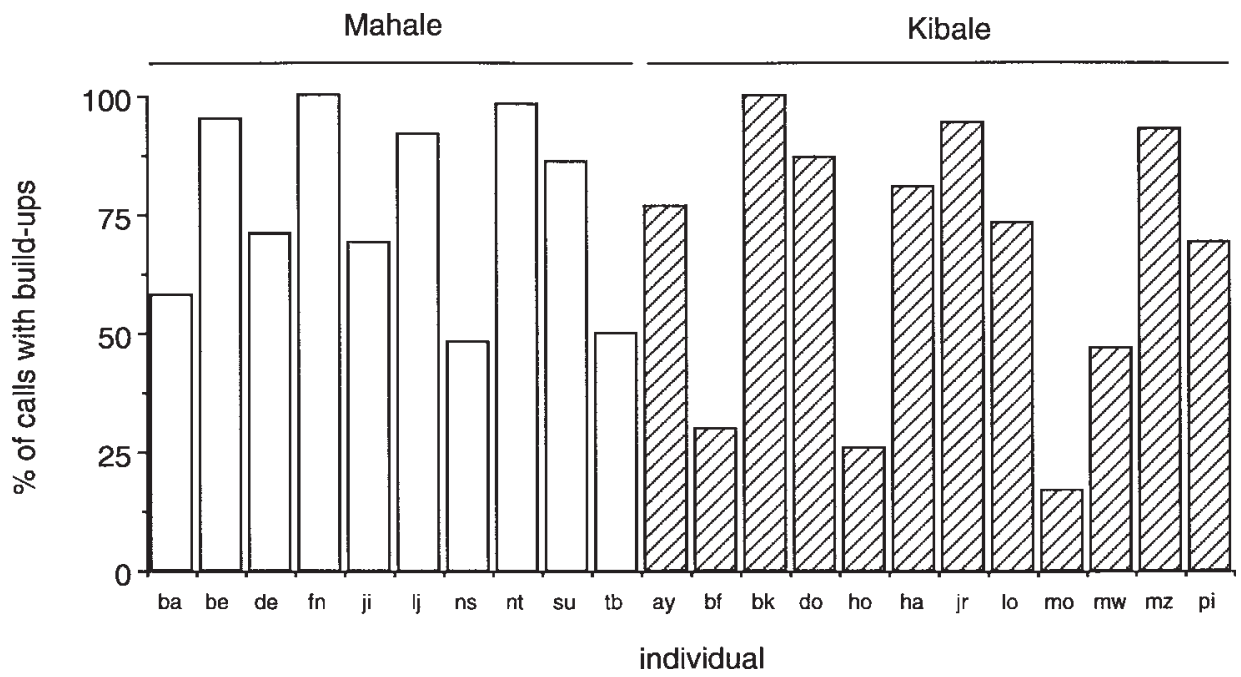

Fig. 4. Production of build-up phases by male chimpanzees from two populations. The mean percentage of calls containing build-ups are shown for each individual from the Mahale and Kibale populations. Open bars, Mahale males. Hatched bars, Kibale males. Sample sizes for each individual are given in Table I.

We employed a discriminant function analysis of build-up phases as a second means to investigate quantitative acoustic differences in the calls produced by males from the two populations. We used the three variables shown to differentiate populations in the nested ANOVA, phase length, rate, and element duration, to generate the discriminant function. These three variables created a function that explained a significant amount of the variation between the two populations (Wilks's lambda $=0.69, \mathrm{~F}_{3,187}=27.77, P<0.001$ ). Each of the three variables showed similarly high canonical loadings, ranging from 0.55 to 0.65 , suggesting that all three contributed equally to the resulting discriminant function. This function classified $78 \%$ of all calls to the correct populations, significantly more than the 50\% expected by chance $\left(\chi^{2}=55.36,1 \mathrm{df}, P<0.001\right.$; Table IIIa). We withheld a subsample of approximately one half of all calls to cross-validate the results of the discriminant analysis. The discriminant function correctly classified $80 \%$ of all calls recorded from the two populations. This classification success rate was again significantly more than that expected based on random assignment $\left(\chi^{2}=63.98,1 \mathrm{df}, P<0.001\right.$; Table IIIb).

\section{Comparisons of Climax Elements}

Nested ANOVA failed to reveal any population differences in climax elements, though three of the seven measured acoustic variables showed marginally significant differences at $P<0.05$ (Table IV). Table $\mathrm{V}$ shows variance components attributable to populations, individuals within populations, and calls within individuals for the acoustic variables measured from climax elements.

Although climax elements produced by males from Mahale and Kibale did not differ significantly in measures of single acoustic variables, these variables could be used in combination to yield readily distinguishable calls by population. A discriminant function employing the three acoustic variables that showed marginally significant differences (Tables IV and V) explained a significant amount of variation between the two populations (Wilks's lambda $=0.83, \mathrm{~F}_{3,246}=17.27, P$ 
Geographic Variation in Chimpanzee Calls / 143

a.

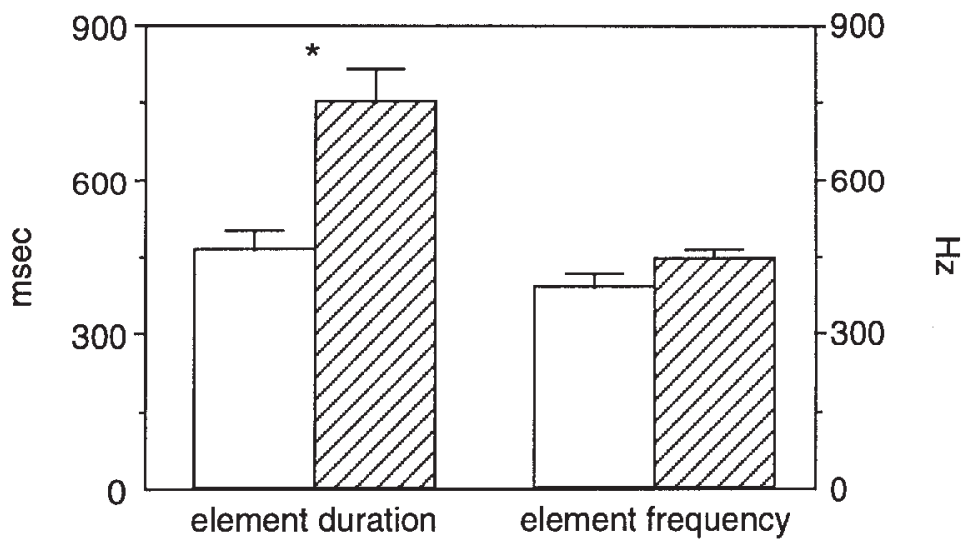

b.

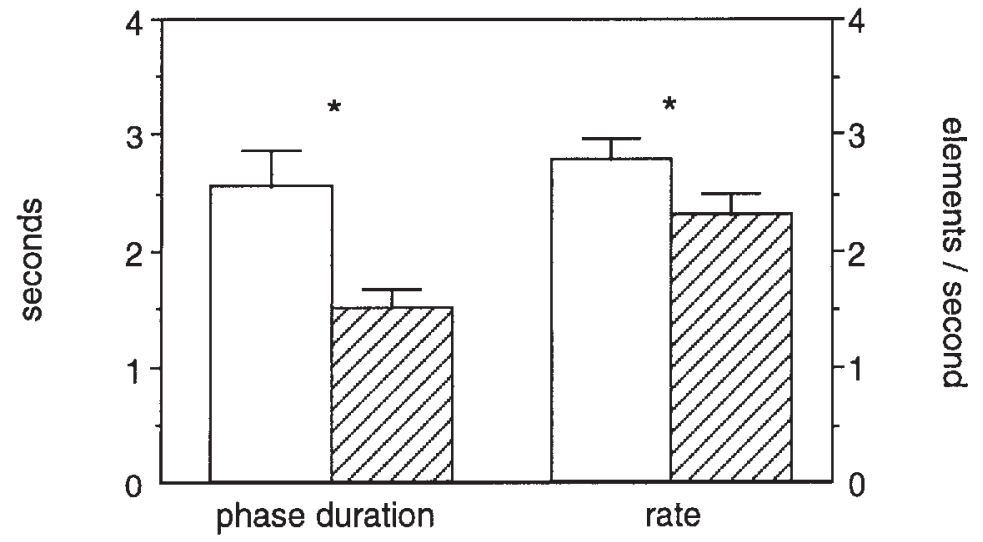

C.

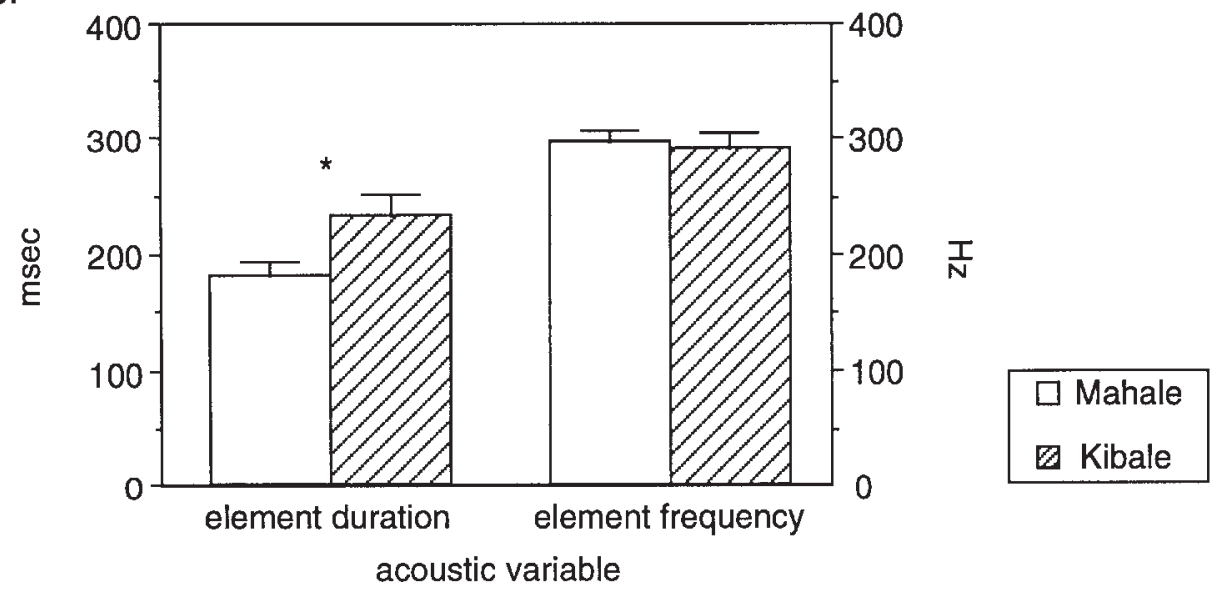

Fig. 5. Acoustic differences between introduction and build-up phases of pant hoots produced by males from Mahale and Kibale. a: Introduction phase. b,c: Build-up phase. Averages of individual means within each population are displayed with 1 se. Between population comparisons marked with asterisks $(*)$ are significant at the sequential Bonferroni criterion. See text for further explanation. 


\section{4 / Mitani et al.}

TABLE II. Variance Components Due to Populations, Individuals Within Populations, and Calls Within Individuals for Six Acoustic Variables Measured From Pant Hoot Introductions and Build-ups

\begin{tabular}{lccc}
\hline & \multicolumn{3}{c}{ \% variance due to } \\
\cline { 2 - 4 } Acoustic variable & Populations & Individuals & Calls \\
\hline Introduction & $40^{*}$ & & 42 \\
1. Element duration & 4 & $18^{*}$ & 51 \\
2. Element frequency & & $45^{*}$ & \\
Build-up & $19^{*}$ & $37^{*}$ & 44 \\
3. Phase duration & $15^{*}$ & $20^{*}$ & 65 \\
4. Rate & $17^{*}$ & $15^{*}$ & 68 \\
5. Element duration & 0 & $14^{*}$ & 86 \\
6. Element frequency & &
\end{tabular}

$* P<$ sequential Bonferroni alpha level.

$<0.001$ ). Using this function, $72 \%$ of all calls were classified correctly according to population (Table VIa), a significantly higher success rate than the $50 \%$ predicted by chance $\left(\chi^{2}=38.71,1 \mathrm{df}, P<0.001\right)$. An additional sample of 270 calls withheld from generating the discriminant function provided cross-validation by assigning $68 \%$ of all calls to the correct population (Table VIb; $\chi^{2}=32.38,1 \mathrm{df}, P$ $<0.001$ ). An examination of the canonical loadings indicates that the discrimination process relied primarily on the average and maximum frequencies of calls; these variables showed relatively high loadings at 0.96 and 0.80 , respectively.

\section{Variability between Populations}

The variability profile based on the coefficients of variation from the 13 acoustic variables illustrates that the calls produced by Mahale chimpanzees are more variable than those made by Kibale males (Fig. $6, \mathrm{t}=2.27 \mathrm{df}=12, P<0.05$ ). This difference is due to the Mahale males producing consistently more variable introduction and climax phases than Kibale individuals (Fig. 6).

\section{DISCUSSION}

The preceding results indicate that the calls produced by male chimpanzees living in two widely separated geographic populations differ in readily discern-

TABLE III. Classification Results of a Discriminant Function Analysis Using Calls From Mahale and Kibale Males*

\begin{tabular}{lccc}
\hline & \multicolumn{3}{c}{ Predicted group } \\
\cline { 2 - 4 } Observed group & Mahale & Kibale & \% correct assignments \\
\hline \multirow{2}{*}{ a) Mahale } & 53 & 24 & $69 \%$ \\
Kibale & 18 & 96 & $84 \%$ \\
Total & 71 & 120 & 191 \\
b) Mahale & 60 & 23 & $72 \%$ \\
Kibale & 14 & 86 & $86 \%$ \\
Total & 74 & 109 & 183 \\
\hline
\end{tabular}

*a) Classification results based on the discriminant function ( $\mathrm{n}=191$ calls); b) Cross-validation classification results based on a sub-sample of calls $(n=183)$ withheld from generating the discriminant function in a). 
Geographic Variation in Chimpanzee Calls / 145

TABLE IV. Acoustic Measurements of Climax Phase Elements ${ }^{\dagger}$

\begin{tabular}{lcr}
\hline Acoustic variable & Mahale & Kibale \\
\hline Duration (msec) & $708 \pm 40$ & $755 \pm 39$ \\
Minimum frequency (Hz) & $701 \pm 46$ & $635 \pm 32$ \\
Maximum frequency (Hz)* & $1,643 \pm 51$ & $1,423 \pm 43$ \\
Frequency range (Hz) & $941 \pm 51$ & $787 \pm 40$ \\
Average frequency $(\mathrm{Hz})^{* *}$ & $1,334 \pm 39$ & $1,134 \pm 34$ \\
F1/F2* & $0.89 \pm 0.05$ & $0.79 \pm 0.05$ \\
Bandwidth $(\mathrm{Hz})$ & $118 \pm 19$ & $111 \pm 19$ \\
\hline
\end{tabular}

The average of individiual means from each populations are shown \pm 1 se.

$* \mathrm{P}<0.05$.

$* * \mathrm{P}<0.025$

ible ways. Males at Kibale begin their calls with significantly longer elements and build-up over shorter periods at slower rates than individuals from the Mahale Mountains. Males from the two populations do not give climax elements that differ significantly in any single acoustic feature. Kibale males, however, show trends of delivering climax elements with more energy concentrated in the lower part of the frequency spectrum and at a lower maximum and average frequencies than males from Mahale. These factors combine to yield discriminable differences in the climax elements produced by males from the two populations. In addition to these differences in mean values, the calls produced by males from the two populations also differ in their overall variability; Mahale chimpanzees deliver appreciably more variable calls than Kibale individuals.

We could not corroborate Clark Arcadi's [1996] earlier claim that the calls given by males from these populations differ qualitatively. Specifically, Kibale males do not consistently give calls that lack a build-up as previously hypothesized. Two reasons, one methodological and the other empirical may underlie these discrepant findings. First, as we noted earlier, Clark Arcadi's analysis was based on relatively small samples from Kibale males. One male in particular, SY, showed a marked tendency to deliver calls without build-ups but contributed a disproportionate share of calls [Clark Arcadi, 1996, Fig. 2]. Thus, the apparent lack of pant hoots containing build-ups that Clark Arcadi described for this population may be attributable to sampling error. Second, and more intriguing is the

TABLE V. Variance Components Due to Populations, Individuals Within Populations, and Calls Within Individuals for Seven Acoustic Variables Measured

From Climax Elements

\begin{tabular}{lccc}
\hline & \multicolumn{3}{c}{$\%$ variance due to } \\
\cline { 2 - 4 } Acoustic variable & Populations & Individuals & Calls \\
\hline & 0 & $28^{*}$ & 72 \\
7. Duration & 0 & $16^{*}$ & 84 \\
8. Minimum frequency & $14^{* *}$ & $35^{*}$ & 51 \\
9. Maximum frequency & 9 & $33^{*}$ & 58 \\
10. Frequency range & $19^{* *}$ & $33^{*}$ & 48 \\
11. Average frequency & $5^{* *}$ & $8^{*}$ & 87 \\
12. F1/F2 & 0 & $19^{*}$ & 81 \\
13. Bandwidth & & &
\end{tabular}

\footnotetext{
$* \mathrm{P}<$ sequential Bonferroni alpha level.
}

$* * \mathrm{P}<0.05$. 


\section{6 / Mitani et al.}

TABLE VI. Classification Results of a Discriminant Function Analysis Using Climax Elements Produced by Mahale and Kibale Males*

\begin{tabular}{lccc}
\hline & \multicolumn{3}{c}{ Predicted group } \\
\cline { 2 - 4 } Observed group & Mahale & Kibale & \% correct assignments \\
\hline a) Mahale & 58 & 36 & $62 \%$ \\
Kibale & 35 & 121 & $78 \%$ \\
Total & 93 & 157 & 250 \\
b) Mahale & 70 & 46 & $60 \%$ \\
Kibale & 40 & 114 & $74 \%$ \\
Total & 110 & 160 & 270 \\
\hline
\end{tabular}

*a) Classification results based on the discriminant function ( $\mathrm{n}=250$ calls); b) Cross-validation classification results based on a sub-sample of calls $(n=270)$ withheld from generating the discriminant function in a).

possibility that the reported differences are real. Clark Arcadi's [1996] sample was derived from the Kanyawara chimpanzee community at Kibale [IsabiryeBasuta, 1988; Wrangham, 1996], which occupies a range approximately $10 \mathrm{~km}$ northwest of the Ngogo community recorded by us. We are currently investigating whether the calls given by males living in these two neighboring populations differ acoustically (Clark Arcadi, Mitani, \& Wrangham, unpublished data).

Earlier reports ascribed geographic differences in chimpanzee calls to a hypothesized role of learning in the vocal acquisition process [Mitani et al., 1992; Mitani \& Brandt, 1994; Clark Arcadi, 1996]. While learning is logically implicated when vocal variations exist between neighboring populations living within dispersal distance, it is less likely to apply to macrogeographic differences between widely separated populations [Marler, 1960; Nottebohm, 1969; Krebs \& Kroodsma, 1980; Mundinger, 1982]. The degree of genetic differentiation between

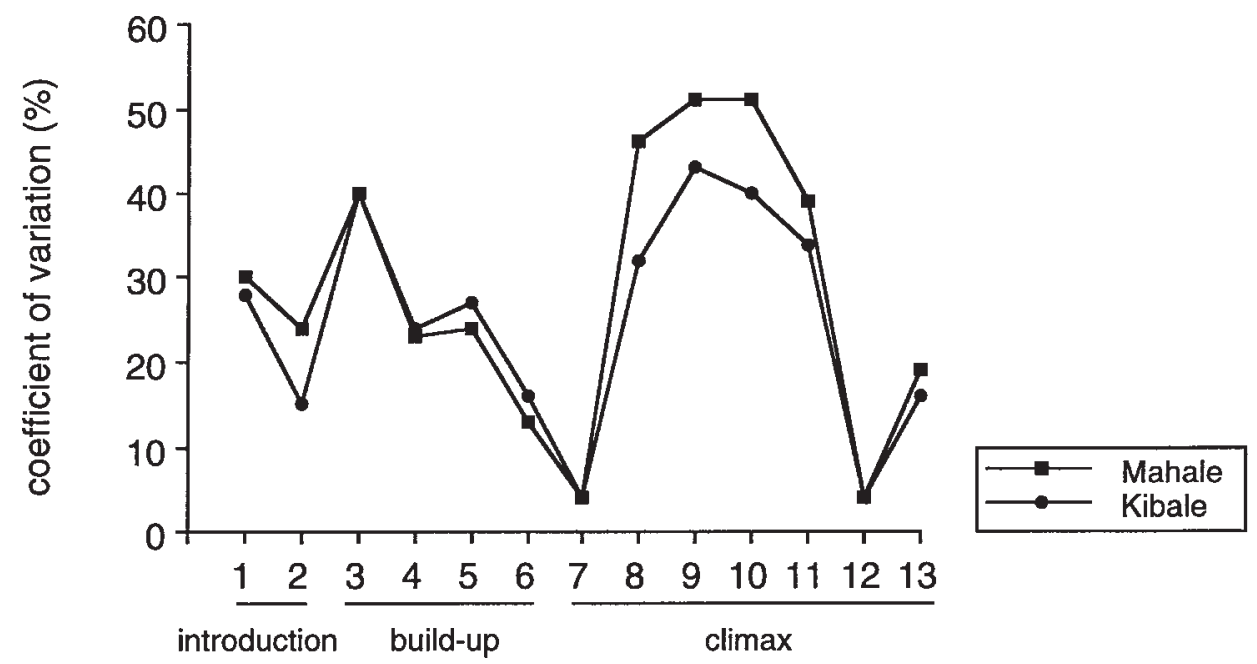

Fig. 6. Variability profile of 13 acoustic variables from pant hoots produced by males from two populations. Mean coefficients of variation for ten males from Mahale and 12 males from Kibale are plotted. Numbers of acoustic variables from each section of the call correspond to those labeled in Tables II and V. 


\section{Geographic Variation in Chimpanzee Calls / 147}

populations is central to the distinction between local and regional variation in vocalizations. Recent studies indicate that chimpanzees are highly vagile and that substantial gene flow over long distances has taken place between populations historically [Morin et al., 1994; Goldberg \& Ruvolo, 1997a,b]. Gene flow appears to be clinally limited by distance, however, with isolation by distance occurring between populations separated by $600 \mathrm{~km}$ or more [Morin et al., 1994; Goldberg \& Ruvolo, 1997a]. The Mahale and Kibale populations are approximately $700 \mathrm{~km}$ apart, and the currently available, though limited, data indicate that they may be genetically isolated [Morin et al., 1994].

The preceding observations suggest that genetically based rather than cultural [sensu McGrew, 1992] differences are likely to underlie any vocal variations that exist between the Mahale and Kibale chimpanzees. If the vocal differences reported here do not reflect learned dialectal variation, to what can we attribute them? Here we propose three, nonmutually exclusive hypotheses.

\section{Habitat Acoustics}

Two hypotheses relate directly to the function of pant hoots in long-distance communication. Field observations suggest that males direct pant hoots to conspecifics who may be several hundred meters away, either to recruit and maintain the company of allies and close associates or possibly to signal status [Mitani \& Nishida, 1993; Clark \& Wrangham, 1994]. The typical usage of these calls makes them particularly susceptible to degradation due to reverberation and frequency-dependent attenuation [Wiley \& Richards, 1982]. The manner in which reverberation and attenuation affect sound transmission, in turn, varies as a function of habitat. Reverberation is especially severe in forests due to the increased potential for reflection and scattering of sound waves off of multiple surfaces, and as a result, calls delivered at relatively slow rates are subject to less degradation than those repeated faster in these habitats [Nottebohm, 1975; Hunter \& Krebs, 1979; Richards \& Wiley, 1980; Anderson \& Conner, 1985; Wiley, 1991]. Attenuation also affects the long-distance transmission of sound, but is less likely to be affected by habitat differences than degradation [Wiley \& Richards, 1982]. Both factors increase monotonically with sound frequency irrespective of habitat. Attenuation, however, increases more quickly in forests compared with open areas, an effect that is again due to scattering. This steeper frequency-dependence of attenuation in forests should typically result in a lower upper limit of the band of acceptable frequencies in forests compared with open habitats. These observations lead to the prediction that animals in forests should produce sounds at lower maximum frequencies than individuals occupying more open areas [Wiley, 1991].

Taken together, the preceding considerations suggest that animals living in forests should deliver relatively low-frequency sounds at slow repetition rates to reduce the effects of degradation and attenuation [Wiley \& Richards, 1982]. These predictions regarding the effects of habitat acoustics on signal form appear to fit well with the observed patterns of macrogeographic variations in chimpanzee calls. The habitats occupied by chimpanzees at Mahale and Kibale differ significantly. Although the Kasoje study site at Mahale is largely forested, the range of the M-group chimpanzees consists of large tracts of woodland, secondary forest, and regenerating farmland [Collins \& McGrew, 1988]. In contrast, the Ngogo study site at Kibale comprises primary rainforest [Ghiglieri, 1984]. Tree enumerations reveal that the Ngogo study area is more densely forested than the Kasoje site. Butynski [1990] counted 404 trees $>10 \mathrm{~cm}$ diameter at breast height (dbh) per ha at Ngogo, while the median number of trees $>7.5 \mathrm{~cm}$ dbh across seven different habitats at Kasoje was only 274 


\section{8 / Mitani et al.}

[Collins \& McGrew, 1988]. Given the more forested area occupied by the Ngogo chimpanzees, selection may have favored the delivery of longer elements given at slower rates to minimize the effects of degradation. Moreover, the three marginally significant geographic differences in the climax phase are consistent with the hypothesis that local habitat acoustics influence variation in signal form. Ngogo males minimize the degradation and attenuation of their calls by delivering elements with slightly more energy concentrated in the lower part of the frequency spectrum and at lower average and maximum frequencies than the Mahale chimpanzees.

\section{Sound Environment}

A second hypothesis is also related to the use of pant hoots as long-distance signals. Long-distance communication is inherently noisy [Wiley, 1983, 1994], a fact due in part to species-rich environments [Marler, 1957]. Given a noisy channel for communication, increased stereotypy of signals may facilitate their detection and recognition over long distances [Marler, 1973; Wiley, 1983, 1994]. The Kibale forest is well known as a site harboring one of the highest population densities of primates anywhere in the world [Struhsaker, 1997]. Seven diurnal primate species live sympatrically with chimpanzees at Ngogo, with primate group densities estimated at 12 groups $/ \mathrm{km}^{2}$ [Butynski, 1990; Struhsaker, Mitani, \& Lwanga, unpublished data]. In contrast, a slightly less diverse set of five diurnal primate species is found at Mahale living at considerably lower densities of approximately seven groups $/ \mathrm{km}^{2}$ (Rosenberg \& Mitani, unpublished observations). In addition, Ngogo contains a rich and diverse avifauna [Struhsaker, 1997] and is home to a more speciose fauna than that found at Mahale [Nishida, 1990]. The high species diversity and density at Ngogo undoubtedly creates a relatively noisy channel for long distance communication and may have led to the greater degree of stereotypy found in the calls produced by Ngogo chimpanzees compared with Mahale males. Measurements of ambient noise levels at both sites will be required to test this hypothesis.

\section{Body Size}

Finally, we suggest that anatomical factors might account for some of the observed geographic variations in chimpanzee calls. Callers radiate sound energy most efficiently at frequencies near their characteristic resonant frequencies. In general, these resonant frequencies will vary inversely with the linear dimensions of the resonator [Kinsler \& Frey, 1962], leading to the prediction that small animals will produce higher frequency sounds than large animals. The strong negative relationship between body size and call frequency found in some primate species is consistent with this prediction [Inoue, 1988; Gouzoules \& Gouzoules, 1990], as are the associations between size and call frequencies documented in other taxa, e.g. anurans and birds [Davies, 1978; Wiley, 1991]. Currently available, though limited, information suggests that the Mahale chimpanzees are smaller than those at Kibale [Mahale $\overline{\mathrm{X}}=42 \mathrm{~kg}, \mathrm{sd}=5.4, \mathrm{n}=6$; Kibale $\mathrm{X}=50 \mathrm{~kg}, \mathrm{n}=2$; Uehara \& Nishida, 1987; Kerbis Peterhans et al., 1993]. The relatively low frequency climax elements produced by Kibale males may thus be a simple by-product of differences in body size. The absence of population differences in call frequencies emitted during the introduction and build-up makes this proposal less compelling than explanations provided by the previous two hypotheses.

\section{Concluding Comments}

In summary, ecological factors related to differences in habitat acoustics, the sound environment of the local biota, and body size are likely to be responsible 
Geographic Variation in Chimpanzee Calls / 149

for the observed geographic variations in chimpanzee calls reported here. Determining the relative importance of each of these factors will be difficult since their predicted effects are not independent. For example, signal degradation and attenuation create high noise levels and will thus act in concert with species-rich sound environments to select for signal stereotypy. Moreover, both large body size and calling in heavily forested habitats favor the production of low frequency calls. Further studies of how the calls of other species differ under varying ecological situations will be necessary to clarify the causes and biological significance of geographic variation in primate vocal behavior. Greater understanding of this variability promises to place the interpretation of reported cultural differences in chimpanzee behavior [McGrew, 1992; Wrangham et al., 1994] in a broader biological context.

\section{CONCLUSIONS}

1. Acoustic analyses reveal that male chimpanzees living in two widely separated geographic regions, the Mahale Mountains and Kibale National Parks, produce acoustically distinguishable pant hoots. Pant hoots differ in quantitative acoustic features rather than qualitatively as previously hypothesized.

2. Males from the Kibale area give longer elements during the introduction and build-up phases of the call and build-up over shorter periods and at slower rates than chimpanzees from Mahale. Kibale males also give less variable calls than chimpanzees at Mahale. Climax elements delivered by males from the two populations do not differ in any single acoustic measure, but the calls from each region are readily discernible through discriminant function analysis.

3. Ecological factors related to differences in habitat acoustics, the sound environment of the local biota, or body size are likely to contribute to the observed macrogeographic variations in chimpanzee calling patterns.

\section{ACKNOWLEDGMENTS}

Field work in Tanzania and Uganda was sponsored by the Tanzanian Commission for Science and Technology, Serengeti Wildlife Research Institute, Tanzanian National Parks, Markerere University and Ugandan National Parks.

We are indebted to G. I. Basuta, J. Kasenene, E. Massawe, A. Seki, T. Nishida, and the staffs of the Mahale Mountains National Park and Makerere University Biological Field Station for logistic support. Several friends and colleagues provided field assistance including $\mathrm{M}$. Bunengwa, $\mathrm{H}$. Bunengwa, C. Businge, M. Hamai, M. Hawazi, H. Hayaki, R. Kitopeni, J. Lwanga, A. Magoba, G. Mbabazi, G. Mutabazi, M. Matamura, L. Ndagizi, T. Nishida, A. Tumusiime, S. Uehara, and D. Watts. We thank J. Gros-Louis for help with data analysis and S. Cerchio, J. Gros-Louis, and J. Pepper for discussions and comments on a draft of the manuscript. Research was supported by a NSF Presidential Faculty Fellowship Award SBR-9253590 and grants from the NSF BNS-9021682, Harry Frank Guggenheim Foundation, National Geographic Society to Mitani and from the Japanese Ministry of Education, Science, and Culture to Professor T. Nishida.

\section{REFERENCES}

Anderson M, Conner R. 1985. Northern cardinal song in three forest habitats in eastern Texas. Wilson Bull 97:436-449.

Beeman K. 1996. Signal software manual. Belmont, MA: Engineering Design.
Bowman R. 1979. Adaptive morphology of song dialects in Darwin's finches. J Ornithol 120:353-359.

Butynski T. 1990. Comparative ecology of blue monkeys (Cercopithecus mitis) in 


\section{0 / Mitani et al.}

high- and low-density subpopulations. Ecol Monographs 60:1-26.

Clark A, Wrangham R. 1994. Chimpanzee arrival pant hoots: Do they signify food or status? Int J Primatol 15:185-206.

Clark Arcadi A. 1996. Phrase structure of wild chimpanzee pant hoots: patterns of production and interpopulation variability. Am J Primatol 29:159-178.

Collins DA, McGrew W. 1988. Habitats of three groups of chimpanzees (Pan troglodytes) in western Tanzania compared. J Hum Evol 17:553-574.

Conner D. 1982. Dialects vs. geographic variation in mammalian vocalizations. Animal Behav 30:297-298.

Darwin C. 1859 . On the origin of species. London: John Murray.

Davies N. 1978. Deep croaks and fighting assessment in toads Bufo bufo. Nature 274:683-685.

Dunn H. 1961. Methods of measuring vowel formant bandwidths. J Acoust Soc Am 33:1737-1746.

Ghiglieri M. 1984. The chimpanzees of the Kibale forest. New York: Columbia University Press.

Goldberg T, Ruvolo M. 1997a. The geographic apportionment of mitochondrial genetic diversity in East African chimpanzees, Pan troglodytes schweinfurthii. Molec Biol Evol 14:976-984.

Goldberg T, Ruvolo M. 1997b. Molecular phylogenetics and historical biogeography of east African chimpanzees. Biol J Linnean Soc 61:301-324.

Goodall J. 1986. The chimpanzees of Gombe. Cambridge: Belknap Press.

Gouzoules H, Gouzoules S. 1990. Body size effects on the acoustic structure of pigtail macaque (Macaca nemestrina) screams. Ethology 85:324-334.

Gouzoules S, Gouzoules H, Marler P. 1984 Rhesus monkey (Macaca mulatta) screams: representational signaling in the recruitment of agonistic aid. Animal Behav 37:182-193.

Green S. 1975. Dialects in Japanese monkeys: Vocal learning and cultural transmission of locale-specific behavior? Zietschrift Tierpsychol 38:304-314.

Hodun A, Snowdon C, Soini P. 1981. Subspecific variation in the long calls of the tamarin, Saguinus fuscicollis. Zietschirft Tierpsychol 57:97-110.

Holm S. 1979. A simple sequentially rejective multiple test procedure. Scandinavian J Stat 6:65-70.

Hunter M, Krebs J. 1979. Geographical variation in the song of the great tit (Paru major) in relation to ecological factors. J Animal Ecol 48:759-785.

Inoue M. 1988. Age gradations in vocalization and body weight in Japanese monkeys (Macaca fuscata). Folia Primatol 51:76-86.
Isabirye-Basuta, G. 1988. Food competition among individuals in a free-ranging chimpanzee community in Kibale Forest, Uganda. Behaviour 105:135-147.

Kerbis-Peterhans J, Wrangham R, Carter M, Hauser M. 1993. A contribution to tropical rainforest taphonomy: retrieval and documentation of chimpanzee remains from Kibale Forest, Uganda. J Hum Evol 25:485-514.

Kinsler L, Frey A. 1962. Fundamentals of acoustics. New York: John Wiley.

Klecka W. 1980. Discriminant analysis. Newbury Park: Sage Publications.

Krebs J, Kroodsma D. 1980. Repertoires and geographical variation in bird song. Advances Study Behav 11:143-177.

Kroodsma D. 1982. Learning and the ontogeny of sound signals in birds. In: Kroodsma $\mathrm{D}$, Miller E, editors. Acoustic communication in birds. New York: Academic Press. Vol 2. $\mathrm{p} 1-23$.

Maeda T, Masataka N. 1987. Locale-specific behavior of the tamarin (Saguinus $l$, labiatus). Ethology 75:25-30.

Marler P. 1957. Specific distinctiveness in the communication signals of birds. Behaviour 11:11-39.

Marler P. 1960. Bird songs and mate selection. In: Lanyon W, Tavolga W, editors. Animal sounds and communication. Washington, DC: American Institute of Biological Sciences. pp 348-367.

Marler P. 1973. A comparison of vocalization of red-tailed monkeys and blue monkeys, Cercopithecus ascanius and $C$. mitis, in Uganda. Zietschrift Tierpsychol 33:233-247.

Marler P, Hobbett L. 1975. Individuality in a long-range vocalization of wild chimpanzees. Zietschrift Tierpsychol 38:97-109.

Marler P, Tamura M. 1964. Culturally transmitted patterns of vocal behavior in sparrows. Science 146:1483-1486.

Marshall J, Marshall E. 1976. Gibbons and their territorial songs. Science 193:235237.

McGrew W. 1992. Chimpanzee material culture. Cambridge: Cambridge University Press.

Mitani J. 1987. Species discrimination of male song in gibbons. Am J Primatol 13:413-423.

Mitani J, Brandt K. 1994. Social factors influence the acoustic variability in the longdistance calls of male chimpanzees. Ethology 96:233-252.

Mitani J, Gros-Louis J, Macedonia J. 1996. Selection for acoustic individuality within the vocal repertoire of wild chimpanzees. Int J Primatol 17:569-583.

Mitani J, Hasegawa T, Gros-Louis J, Marler P, Byrne R. 1992. Dialects in wild chimpanzees? Am J Primatol 27:233-243. 


\section{Geographic Variation in Chimpanzee Calls / 151}

Mitani J, Nishida T. 1993. Contexts and social correlates of long distance calling by male chimpanzees. Animal Behav 45:735-746.

Morin PA, Moore J, Chakraborty R, Jin L, Goodall J, Woodruff DS. 1994. Kin selection, social structure, gene flow, and the evolution of chimpanzees. Science 265:1193-1201.

Mundinger P. 1982. Microgeographic and macrogeographic variation in acquired vocalizations in birds. In: Kroodsma D, Miller E, editors. Acoustic communication in birds, Vol 2. New York: Academic Press, p 174-208.

Nishida T. 1990. The chimpanzees of the Mahale mountains. Tokyo: Tokyo University Press.

Nishida T, Takasaki H, Takahata Y. 1990. Demography and reproductive profiles. In Nishida T, editor. The chimpanzees of the Mahale mountains. Tokyo: University of Tokyo Press. p 63-97.

Nottebohm F. 1969. The song of the chingolo, Zonotrichia capensis, in Argentina: description and evaluation of a system of dialects. Condor 71:299-315.

Nottebohm F. 1975. Continental patterns of song variability in Zonotrichia capensis: some possible ecological correlates. Am Natur 109:605-624.

Richards D, Wiley RH. 1980. Reverberations and amplitude fluctuations in the propagation of sound in a forest: implications for animal communication. Am Natur 115:381-399.

Seyfarth R, Cheney D, Marler P. 1980. Monkey responses to three different alarm calls: evidence of predator classification and semantic communication. Science 210:801-803.

Sokal R, Rohlf FJ. 1995. Biometry. New York: W.H. Freeman.

Sokal R, Braumann C. 1980. Significance tests for coefficients of variation and variability profiles. Syst Zool 29:50-66.

Sorjonen J. 1986. Factors affecting the structure of song and the singing behaviour of some northern European passerine birds. Behaviour 98:286-304.

Struhsaker T. 1970. Phylogenetic implica- tions of some vocalizations of Cercopithecus monkeys. In: Napier P, Napier J, editors. Old world monkeys. New York: Academic Press. p 365-444.

Struhsaker T. 1997. Ecology of an African rain forest. Gainesville: University Press of Florida.

Uehara S, Nishida T. 1987. Body weights of wild chimpanzees (Pan troglodytes) of the Mahale Mountains National Park, Tanzania. Am J Phys Anthropol 72:315-321.

Waser P. 1977. Individual recognition, intragroup cohesion and intergroup spacing: evidence from sound playback to forest monkeys. Behaviour 60:28-74.

Wiley RH. 1983. The evolution of communication: information and manipulation. In Animal Behaviour, Vol 2: Communication. Halliday T, Slater PJB, editors. New York: W.H. Freeman. p 156-189.

Wiley RH. 1994. Errors, exaggeration, and deception in animal communication. In: Behavioral mechanisms in evolutionary ecology. Real L, editor. Chicago: University of Chicago Press. p 157-189.

Wiley RH. 1991. Associations of song properties with habitats for territorial oscine birds of eastern North America. Am Natur 138:973-993.

Wiley RH, Richards D. 1982. Adaptations for acoustic communication in birds: sound transmission and signal detection. In: Acoustic communication in birds, vol 1 . Kroodsma DE, Miller EH, editors. New York: Academic Press. p 131-181.

Wilkinson L, Hill M, Vang E. 1992. Systat: statistics, version 5.2. Evanston: Systat, Inc. Wrangham R. 1996. Social ecology of Kanyawara chimpanzees: implications for understanding the costs of great ape groups. In: Great ape societies. McGrew W, Marchant L, Nishida T, editors. Cambridge: Cambridge University Press. p 45-57.

Wrangham R, McGrew W, deWaal F, Heltne P. 1994. Chimpanzee cultures. Cambridge: Harvard University Press.

Zuberbuler K, Noe R, Seyfarth R. 1997. Diana monkey long-distance calls: messages for conspecifics and predators. Animal Behav 53:589-604. 